Review

\title{
T-Cell Receptor-Like Antibodies: Targeting the Intracellular Proteome Therapeutic Potential and Clinical Applications
}

\section{Maya Cohen and Yoram Reiter*}

Faculty of Biology, Technion-Israel Institute of Technology, Haifa 32000, Israel

* Author to whom correspondence should be addressed; E-Mail: reiter@tx.technion.ac.il; Tel.: +972-4-8292785; Fax: +972-4-8293779.

Received: 15 August 2013; in revised form: 22 August 2013/ Accepted: 22 August 2013 / Published: 17 September 2013

\begin{abstract}
Major histocompatibility complex (MHC) class I molecules are key in the immune response against malignant cells by shaping the T-cell repertoire and presenting peptides from endogenous antigens to $\mathrm{CD} 8+$ cytotoxic $\mathrm{T}$ cells. Because of their unique specificity, MHC-peptide complexes are a desirable target for novel immunotherapeutic approaches. These complexes can be targeted by recombinant T-cell receptors (TCRs). However, most TCRs produced thus far have affinities which are too low for target detection under normal assay conditions, and limited stability (due to their generation in a single-chain version). Developing high-affinity soluble antibody molecules endowed with a TCR-like specificity toward tumor epitopes, termed TCR-like antibodies, addresses the low affinity of TCRs. These TCR-like antibodies are being developed as a new immunotherapeutic class for targeting tumor cells and mediating their specific killing. In addition, these antibodies are valuable research reagents enabling the study of human class I peptide-MHC ligand-presentation and TCR-peptide-MHC interactions.
\end{abstract}

Keywords: antibodies; antibody engineering; immunotoxins; MHC-peptide complex; phage display; recombinant antibodies; T-cell receptor; cancer immunotherapy

\section{T-Cell Epitopes as Targets for Immunotherapy}

Cellular expression of specific peptides in a complex with major histocompatibility complex (MHC) class I molecules has been shown to be associated with cancer and autoimmune disorders [1-3]. In cancer, the discovery of these peptides emerged from the observation that human tumor cells 
express antigens that are recognized by cytotoxic T lymphocytes (CTLs) derived from patients [1-5]. Despite the identification of tumor-associated MHC-peptide complexes [6], and the presence of CTLs specific for these complexes in cancer patients [5], tumors were demonstrated to develop mechanisms limiting the activity of the CTLs and the anti-tumor immune response was reported to fail in inducing tumor regression [7-9].

In recent years, the design and development of strategies augmenting active specific immunotherapies in cancer patients advanced significantly due to the discovery of well-defined tumor-associated antigens (TAAs) and the development of a methodology to monitor immune responses [2,3,10,11]. The list of tumor antigens recognized by T-cells is growing in number of antigens as well as in the types of tumor cells that express them. Attractive tumor antigen (T-cell epitopes) targets can be classified into three groups: (i) tumor-specific antigens arising from mutated proteins, such as $\beta$-catenin in colon cancer [12]; (ii) differentiation antigens with an expression that is restricted to a certain cell lineage and is therefore very specific (e.g., the melanoma-specific targets gp100 MART1, tyrosinase antigens expressed in the melanocytes [13], and the testicular cancer antigens such as NY-ESO-1, which are expressed only by tumor cells and spermatogenic cells from the testis) $[14,15]$; and (iii) antigens derived from gene overexpression or amplification (e.g., HER-2/neu) [16,17].

A broad spectrum of immunotherapeutic approaches was developed in an attempt to enhance the adoptive cellular immune response to tumors, including adoptive transfer of $\mathrm{T}$ cells, cancer peptide vaccines, autologous cancer vaccines, and vaccination with dendritic cells (DCs) [18,19]. These approaches are currently in various stages of clinical development $[18,19]$.

Cell-based approaches present challenges such as the existence of regulatory mechanisms that may limit the activity of antitumor CTLs, or the fact that CTLs which are required for the development of some of the aforementioned approaches are relatively rare and may be nonfunctional or anergic. An approach that could potentially overcome some of the challenges of the cell-based approaches is to use antibodies to mimic the fine unique specificity of T cells. Since the specificity of the immune response is regulated and dictated by the unique tumor-associated class I MHC-peptide complexes, it should be possible to use these very specific and unique molecular cell-surface markers as targets to eliminate cancer cells, while sparing the normal cells. In addition, a well-chosen, relatively small panel of reagents with T-cell receptor (TCR)-like specificity directed against different peptide-MHC complexes should allow targeting of the vast majority of the more common tumors in a given population. The rationale of making antibodies with TCR-like specificity lies in the possibility of using the same T-cell based specificity of tumor cell recognition through tumor-associated MHC-peptide complexes that are recognized by $\mathrm{T}$ cells. However, instead of using a cell-based approach, an antibody-based approach is being applied. Soluble antibodies can be produced in large quantities, penetrate tumors better than $\mathrm{T}$ cells, exert biologic activity that is similar to that of $\mathrm{T}$ cells, and be more efficient as a therapeutic modality. Thus, the TCR-like antibody approach combines two main advantages of the immune system: the fine specificity of $\mathrm{T}$ cells with the biological and pharmacological properties of an antibody that is not susceptible to most tumor regulatory influences that limit T cells. Notably, down regulation of MHC presentation by tumors is the most relevant tumor environment regulatory mechanism that can influence the cell-based approach as well as the TCR-like antibody approach. With this limitation in mind, attempts are being made to generate high affinity TCR-like antibodies that are suitable for detection of relatively low-density of MHC-presented antigens. 


\section{T-Cell Receptor-Like Antibodies}

Antibodies with the MHC-restricted specificity of $\mathrm{T}$ cells are rare and have been difficult to generate [20]. TCRs recognize intracellularly processed linear peptide antigens in the context of MHC molecules. This is in sharp contrast to the recognition mode of B cells, which via soluble or membrane antibodies recognize three-dimensional target structures in their cognate antigen without any MHC restriction. Thus, $\mathrm{T}$ and $\mathrm{B}$ cells represent two fundamentally different recognition modes of the specific immune system. Through alternating selection processes, $\mathrm{T}$ cells are educated to recognize antigenic peptides presented in complex with MHC class I or II molecules, whereas B cells are not subjected to this selection. The distinctly different education of $\mathrm{B}$ and $\mathrm{T}$ cells explains why antibodies with the MHC-restricted specificity of $\mathrm{T}$ cells occur rarely and why it has been difficult to generate such specificities using conventional B-cell hybridoma techniques. Soluble and specific reagents for specific MHC-peptide structures include soluble versions of TCRs or antibodies. The last few years have witnessed a major progress in the development of such antibodies, as several groups were able to generate, occasionally in a reproducible manner, TCR-like antibodies directed against a growing repertoire of tumor and viral T-cell epitopes.

The key for successful production of TCR-like antibody (using either conventional hybridoma or phage display technology) is having a purified recombinant MHC-peptide complex folded in a native conformation that is recognized by a T cell [20]. MHC-peptide complexes are generated by expressing the extracellular domains of the human leukocyte antigen (HLA) heavy chain and 2-microglobulin inclusion bodies in E. coli followed by their in-vitro refolding in the presence of the desired HLA-restricted peptide [21,22]. The refolded peptide-MHC complexes are very pure, monomeric, and folded in the right conformation, as demonstrated by structural [23] and functional studies [21,24]. These complexes can be further biotinylated in a site-specific manner, a feature that is utilized for the in-vitro selection procedures of TCR-like antibody isolation and for the subsequent step of specificity characterization. Several laboratories attempted to generate antibodies recognizing T-cell epitopes [20,25-50]; however, many laboratories experienced difficulties and some authors have published their failure to isolate such antibodies [44,45]. A major breakthrough in generating these antibodies involved the development (in the mid-1990s) of alternative strategies to the conventional hybridoma approach. The pioneering work by Andersen and colleagues (1996) demonstrated the use of an antibody phage display approach as a tool for isolating antibodies with a unique specificity [20]. This format of phage display technology consists of generating antibody libraries exposed as fusion proteins on the surface of phage particles. Each phage particle displays a unique antibody (a Fab or single chain [sc] Fv fragment), which is encoded by genes present within the same phage particle. The purification of specific phages is accomplished by repeated rounds of selection on a specific target followed by amplification in bacterial cells of the eluted bound phages. Thus, in contrast to hybridoma technology, the phage display procedure involves positive selection for the requested binder. Several groups have developed methods for generating comprehensive phage display libraries of murine or human antibody Fab or scFv molecule fragments in E. coli cells (for a review see reference [51]).

A phage display approach enabled the isolation of peptide-specific, MHC-restricted antibodies to murine class I complexes at first [20], and subsequently to human disease-related human HLA-peptide complexes using large naïve human phage display libraries [27,29-32,35,37,40,48,52,53]. 


\section{Generation of TCR-like Antibodies}

Antibodies with MHC-restricted specificity of $\mathrm{T}$ cells have been traditionally rare and difficult to generate. However, the last few years saw a major progress in developing such antibodies, as several groups have been able to generate, occasionally in a reproducible manner, TCR-like antibodies directed to a growing repertoire of tumour and viral T-cell epitopes. Immunization strategies, in-vitro selection by phage display, and a combination thereof have been reported for isolating TCR-like antibodies.

Obtaining a purified recombinant $\mathrm{MHC}$ /peptide complex folded in a native conformation that is recognized by a T-cell [20] proved to be key for a successful production of TCR-like antibody using either conventional hybridoma or phage display technology. Recombinant MHC/peptide complexes are efficiently produced in a relatively high amount needed for the purpose of antibody isolation. These complexes are generated by expressing the extracellular domains of the human leucocyte antigen (HLA) heavy chain and 2-microglobulin inclusion bodies in E. coli followed by their in-vitro refolding in the presence of the desired HLA-restricted peptide [21,22]. The refolded peptide/MHC complexes are very pure, monomeric, and folded in the right conformation, as demonstrated by structural [23] and functional studies [21,24]. These complexes can be further biotinylated in a site-specific manner, a feature that is utilized for the in-vitro selection procedures of TCR-like antibody isolation and for the subsequent step of specificity characterization.

\subsection{Phage Display Libraries}

A major breakthrough in making TCR-like antibodies was achieved when alternative strategies to the conventional hybridoma approach were developed in the mid 1990s. The pioneering work by Andersen and colleagues (1996) demonstrated the ability to use the antibody phage display approach as a tool to isolate antibodies with a unique specificity [20]. Phage display technology includes generating antibody libraries exposed as fusion proteins on the surface of phage particles. Each phage particle displays a unique antibody (a Fab or scFv fragment) encoded by genes present within the same phage particle. Phage particles carrying specific antibodies are purified by repeated rounds of selection on a specific target followed by amplification in bacterial cells of the eluted bound phages. Thus, in contrast to the hybridoma technology, the phage display procedure involves positive selection for the requested binder (For a review of phage display see [54]). A phage display approach enabled the isolation of peptide-specific, MHC-restricted antibodies to murine class I complexes, at first [20], and subsequently also to human disease-related human HLA-peptide complexes [27,29-32,35,37,48].

\subsubsection{Naïve Libraries}

TCR-like antibodies were isolated from large naïve human phage display libraries by our group and others [52,53]. Several different antibody clones directed to a single T-cell epitope were isolated, in a reproducible manner, from these libraries. The high frequency and high affinities of some of the isolated antibodies suggest that these molecules may be present in the antibody repertoires from the B-cell donors of the phage library; however, an in vivo role for such antibodies remains unclear. To the best of our knowledge, no evidence for B-cell expression of TCR-like antibodies exists. We believe that while the genetic information of TCR-like antibodies exists in the germ line, these molecules are 
either not produced or are negatively selected. The TCR-like phage-derived Fabs may be the outcome of gene recombination that does not occur naturally. Regardless of the reason for the observed high frequency of antibodies to MHC-peptides, the phage-based approach can be consistently applied to isolate recombinant antibodies with TCR-like specificity to a large variety of MHC-peptide complexes.

The affinity of TCR-like antibodies isolated from a naïve phage display library is not always sufficient for therapeutic purposes. Both random [55] and rational [56] affinity-maturation strategies were developed to increase the affinity of TCR-like antibodies. Chames and colleagues (2000) reported an 18-fold improved affinity of TCR-like Fab directed to the tumour T-cell epitope HLA-A1/MAGE-A1 using randomized in vitro affinity maturation. Fab G8 with affinity of $250 \mathrm{nM}$ was used as the platform for construction of two randomized libraries: L chain shuffling library and $\mathrm{H}$ chain complementarity-determining region (CDR) three mutated library. $A V_{H} / V_{L}$ hybrid (clone Hyb3) constructed from clones isolated from these two libraries had an affinity of $14 \mathrm{nM}$ while keeping the fine specificity of the parental antibody [29].

Rational design for affinity maturation of TCR-like antibodies was demonstrated by Renner and colleagues. They analyzed high-resolution structure of TCR-like Fab 3M4E5 bound to the HLAA2/NY-ESO-1 complex and compared it with the structure of a cognate TCR/pMHC complex. Based on the structural data, they generated a second generation Fab library based on Fab 2M4E5 in which they randomized residues at positions that could optimize peptide interaction without increasing MHC binding. Key residues responsible for direct binding of a peptide motif as well as residues facing the MHC helices were left unchanged. This approach resulted in a 20 -fold affinity improvement of a new isolated TCR-like Fab to the HLA-A*0201/NY-ESO-1 epitope.

TCR-like antibodies isolated from phage display libraries are in a monovalent antibody form (Fab or scFv fragments) and can be produced in bacterial expression systems with relatively high-yields of stable and purified antibody. Monovalent fragments are useful reagents for a variety of TCR-like applications such as epitope presentation [57] and structural [58] studies. In addition, monovalent fragments can be used as targeting agents when conjugated to a cargo protein, (e.g., a truncated form of Pseudomonas exotoxin in the case of immunotoxin) [59]. Notably, one of the limitations of the monovalent antibody form is its reduced binding intensity, which is a major disadvantage when using a TCR-like antibody as a tool to study low-density epitopes (e.g., some tumor-associated MHC-peptide complexes presented by tumor cells). To overcome this limitation, Fab- or scFv-tetramers [60], with directly tagged fluorescent probes, can be generated to increase the binding avidity and consequently the binding intensity of specific MHC-peptide complexes [31]. Another approach developed to overcome the reduced binding intensity involves increasing the avidity of the TCR-like antibody molecules by transforming the monovalent fragments into bivalent constructs in the form of $\operatorname{IgG}$ molecules. The $\mathrm{H}$ and L Fab genes are cloned into IgG antibody vectors carrying secretion signal for expression and secretion in eukaryotic expression systems. This strategy was successfully implemented in our lab for several TCR-like Fabs, and for the most part, resulted in the production of TCR-like IgG molecules which kept the fine specificity of the parental monovalent fragment while significantly improving its detection sensitivity and apparent binding affinity [56,61]. 


\subsubsection{Immunized Libraries}

In the immunized phage display approach, which is a strategy complementary to the aforementioned naïve phage display approach, mice are immunized with the desired recombinant $\mathrm{MHC} /$ peptide complexes and phage display libraries are constructed from the spleens of these mice $[39,62]$. In a syngenic immunization approach, transgenic mice, which express the desired human HLA allele on a murine MHC knocked out background, are immunized with the recombinant MHC/peptide complexes. The use of specific HLA allele transgenic mice, that have self tolerance to their transgenic HLA allele, increases the probability of isolating a rare TCR-like antibody rather than pan-reactive anti-HLA antibody with no peptide specificity.

\subsection{Hybridoma Technology}

The effectiveness of the immunization protocol used is key for obtaining TCR-like antibodies using either a phage display approach with immunized libraries or hybridoma technology. The earliest published studies on hybridoma technology and optimized immunization and screening strategies, used antigen presenting cells (APCs) presenting strongly immunogenic peptides in their MHC complex as immunogenes [33,42]. It was reported that one to three out of approximately 1,000 growth-positive clones tested produced antibodies of the requested specificity, illustrating that peptide-specific, MHC-restricted antibodies are quite rare even under optimal conditions of induction and underscoring why many attempts have failed [44,45,63]. More recently, several groups have been successful in using a conventional hybridoma approach for isolating TCR-like antibodies directed to tumour [63,64] and viral [65] epitopes. These groups used recombinant MHC/peptide complexes for the immunization and high throughput screening of a few thousands of clones for the isolation of rare TCR-like antibodies [63-65].

\subsection{Phage Display vs. Hybridoma-Derived Antibodies: Pros and Cons}

The investigator's resources and field of expertise should determine the method of choice for isolating TCR-like antibodies. The most common approach for such isolation published to date is large phage display libraries [66]. The major advantage of this approach is the power of high selection toward the desired antigen that is achieved within a relatively short procedure. Other advantages include efficient and reproducible isolation of antibodies directed to different T-cell epitopes from the same source. In contrast, generation of hybridomas for isolation of antibodies with such defined antigenic determinant within the immunogen is somewhat less efficient, and a relatively longer procedure is required for isolation of rare clones. Furthermore, isolation of TCR-like antibodies using hybridoma methodology may be limited to stable MHC complexes which bind the peptide with high affinity [67], as reduced stability/half-life of MHC/low-affinity-peptide complexes might not induce effective immunization and in vivo IgG maturation.

Advantages of the hybridoma technology include a tendency for isolating antibodies with highaffinity binding to the MHC/peptide complex, as in this approach, the antibodies produced are the result of multiple antigen challenges leading to in vivo affinity maturation of the antibody clones before their isolation. TCR-like antibodies isolated using hybridoma technology were reported to have 
relatively high binding affinity (low nanomolar range) $[63,68]$ compared with the moderate average affinity $(\sim 50-300 \mathrm{nM})$ of phage-derived TCR-like antibodies isolated from naïve libraries [56,61]. Notably, although rare and technically challenging, few antibodies in the low nanomolar range were successfully isolated from a naïve phage display library using various screening and selection techniques [69]. Furthermore, technological improvements such as the aforementioned second generation libraries and in vitro affinity maturation procedures improve binding affinities to levels comparable to those observed with hybridoma technology.

TCR-like antibodies isolated from phage display libraries are in a monovalent antibody form (Fab or scFv fragments) and can be produced in bacterial expression systems with relatively highyields of stable and purified antibody. Monovalent fragments are useful reagents for a variety of TCRlike applications such as epitope presentation [57] and structural [58] studies. In addition, monovalent fragments can be used as targeting agents when conjugated to a cargo protein, (e.g., a truncated form of Pseudomonas exotoxin in the case of immunotoxin) [59]. Notably, one of the limitations of the monovalent antibody form is its reduced binding intensity, which is a major disadvantage when using a TCR-like antibody as a tool to study low-density epitopes (e.g., some tumour-associated MHC/peptide complexes presented by tumour cells). To overcome this limitation, Fab/scFv-tetramers [60], with directly tagged fluorescent probes, can be generated to increase the binding avidity, and consequently, the binding intensity of specific MHC/peptide complexes [31]. Fab tetramers are generated by introducing a BirA sequence tag for site-specific biotinylation at the C-terminus of the Fab's CL or $\mathrm{CH} 1$ domain for tetramerization by labelled streptavidin reagent. Another approach developed to overcome the reduced binding intensity involves increasing the avidity of the TCR-like antibody molecules by transforming the monovalent fragments into bivalent constructs in the form of $\operatorname{IgG}$ molecules. The $\mathrm{H}$ and L Fab genes are cloned into IgG antibody vectors carrying secretion signal for expression and secretion in eukaryotic expression systems. This strategy was successfully implemented in our lab for several TCR-like Fabs, and for the most part, resulted in the production of TCR-like IgG molecules which kept the fine specificity of the parental monovalent fragment while improving its detection sensitivity and apparent binding affinity significantly [61,57]. The Fc portion of the IgG is essential when using TCR-like antibodies to target cells for cytotoxic effects by recruiting components of the immune systems through antibody-dependent-cell mediated cytotoxicity (ADCC) or complement-dependent-cytotoxicity (CDC). The clinical development of TCR-like IgG antibodies will require humanisation of the mouse IgG hybridoma clones to avoid anti-idiotypic host response and to optimize the effector functions and half-life of the antibody.

\section{TCR-Like Antibodies as Therapeutic Agents}

The development of antibody-based therapeutics has been rapid since the hybridoma technology was first established in 1975 by Kohler and Milstein. Today, anticancer monoclonal antibodies (mAbs) are a growing family of novel molecules applied in the treatment regimens for solid and hematological cancers. The long half-life, low toxicity, and high affinity and specificity of mAbs are only a few of the advantages that make them attractive therapeutic agents [70]. Over 10 antibody-based anti-cancer immunotherapeutic approaches have received regulatory approval and many more are under clinical development. Antibody-based immunotherapeutic approaches include the use of naked antibodies as 
well as antibody conjugates (e.g., immunotoxins, fusion with cytokine molecules, immunoradioisotope labeling, and bi-specific antibody therapy).

Presentation of specific MHC-peptide complexes associated with a certain tumor type supports the use of TCR-like antibodies as specific anti-tumor targeting molecules. Whereas therapeutic anti-cancer mAbs target membrane proteins, TCR-like antibodies expand the targeting repertoire to the intracellular proteome represented by the MHC surveillance mechanism of the cell. Such specific antibodies present new opportunities for anti-cancer targeting through the transformation of the fine cellular specificity of T-cell recognition into an antibody-based immunotherapeutic approach [71].

\subsection{Naked TCR-Like Antibodies}

Recruitment of innate immunity is among the proposed mechanism for unconjugated anti-tumorspecific mAbs such as rituximab (Rituxan/MabThera, Genentech Inc, South San Francisco, CA, USA) [72] and trastuzumab (Herceptin, Genentech Inc, South San Francisco, CA, USA) [73]. Similar to mAbs that target a specific tumor antigen, TCR-like antibodies are being used to mediate antibody-dependent cell-mediated cytotoxicity (ADCC) and complement-dependent cytotoxicity (CDC). Weidanz and colleagues (2006) generated a mouse IgG2a mAb RL4B specific to human chorionic gonadotropin (hCG)- $\beta$ presented by HLA-A2 [63] and demonstrated that this mAb prevented and suppressed breast tumor growth in xenograft in-vivo models [68]. As increased treatment efficiencies were recently reported for mAbs when used in combination with other treatment modalities (e.g., in first-line treatment of metastatic colorectal cancer, both US and European guidelines recommend a $\mathrm{mAb} /$ chemotherapy combination) [74], in future, TCR-like antibodies could be evaluated in combination with other treatment modalities such as other immunotherapies or chemotherapeutic agents.

\subsection{Immunotoxins}

An immunotoxin is a chimeric protein consisting of a targeting moiety linked to a toxin used to kill the diseased cell while sparing the normal cell. The target moiety can be an antibody or a ligand directed against a receptor or cell surface antigen specific to the disease. The toxin can be any molecule that will induce cell death by directly interacting with molecules involved in cellular processes, by modifying the cell membrane, or by inducing apoptotic proteins thereby causing cell death indirectly.

The first immunotoxins were produced by chemically coupling native toxins to antibodies with reagents that form disulfide bonds connecting the toxin to the antibody. These first-generation immunotoxins lacked specificity and were characterized by immunogenicity, poor stability, and heterogeneous composition. The second generation of immunotoxins was generated after research and crystallographic studies of the first-generation immunotoxins. These immunotoxins were still produced by chemical conjugation; however, the non-specific cell-binding domain of the toxin was removed and replaced by an antibody. These second-generation immunotoxins were more specific and consequently safer, thereby facilitating the use of increased dosages in animal models. These constructs were still heterogeneous (due to the chemical conjugation), and their ability to penetrate solid tumors was poor (due to their size). Third-generation immunotoxins are made by recombinant DNA techniques whereby the DNA encoding the cell binding domain of the toxin is replaced by DNA sequences 
encoding only the antigen-binding site of the antibody (the variable fragment [Fv] portion). Recombinant immunotoxins are homogeneous, with improved tumor penetration, and are relatively inexpensive to produce [75-78].

Bacteria and plants produce highly potent toxins which are among the most powerful cytotoxic agents in nature. These toxins must be produced in microorganisms (typically, E. coli) because they kill animal cells. Bacterial and plant-derived toxins have been chemically conjugated or genetically fused to different ligands that target cancer cells. Currently, most of the recombinant immunotoxins being evaluated in clinical trials use either diphtheria toxin (DT) or Pseudomonas exotoxin A (PE), because these bacterial toxins are more easily produced in $E$ coli than plant toxins and because they have demonstrated more activity and fewer side effects in humans [78].

Immunotoxins are designed to bind specific antigens on the surface of cancer cells and enter the cells by endocytosis that carries them into the endosomal compartment via clathrin coated pits $[79,80]$. Subsequently, the toxins are processed by furin cleavage to produce two fragments that are initially held together by a disulfide bond [81,82]. After translocation to the cytosol (with different translocation mechanisms for PE and DT) the enzymatically active fragment of the toxin catalyzes adenine diphosphate (ADP) ribosylation of a diphthamide residue of elongation factor 2 (EF2) $[83,84]$, thereby causing arrest of protein synthesis, leading to apoptotic cell death. A truncated form of PE (38 kDa) that is frequently used is called PE38. This form contains PE amino acids 253-364 and 381-613 [85], and does not include the binding domain of PE, which binds to normal cells. PE kills cells by catalyzing the irreversible ADP-ribosylation and subsequent inactivation of elongation factor 2 (EF-2), using nicotinamide adenine dinucleotide $\left(\mathrm{NAD}^{+}\right)$as a factor. As a consequence, protein synthesis is inhibited and cell death ensues. Notably, mutating the carboxyl-terminal sequence of PE from REDLK to KDEL could be useful as this mutation improves the cytotoxicity of PE and its derivatives, presumably by improving their delivery to the endoplasmic reticulum where translocation to the cytosol takes place [86]. At present, no antibody-based immunotoxin has been approved by the US food and drug administration (FDA). However a recombinant toxin that contains the human interleukin-2 (IL-2) and truncated DT (denileukin diftitox [Ontak ${ }^{\circledR}$ ], Eisai Inc., Woodcliff Lake, NJ, USA) is approved for use in cutaneous T-cell lymphoma (CTCL). Additionally, an anti-CD22 Fv immunotoxin that contains the truncated PE38 form has shown satisfying results in the treatment of hairy cell leukemia (HCL). Antibody-based immunotoxins are also associated with side effect and toxicities, which have restricted the administered dose. Further research will likely improve their safety profile thereby facilitating a broader clinical use in cancer treatment.

\subsection{TCR-Like Antibody-Toxin Fusion Molecule}

TCR-like antibodies could potentially be used to deliver the truncated form of the mutated PE (PE38KDEL) into target tumor cells. In our lab, we generated fusion molecules in which the different TCR-like antibody Fabs or scFv were fused to PE38KDEL, by fusing the truncated PE38KDEL gene at its N-terminus to the C-terminus of each Fab light chain (CL) as shown schematically in Figure 1. TCR-like immunotoxins have also been generated against HLA-A2 positive melanoma differentiation antigens gp100 [87] and MART-1 [59], and against the prostate and breast tumor-associated antigen TRAP [88]. 
The TCR-like Fab-PE38KDEL fusion molecules maintained their ability to bind the authentic endogenously-derived MHC-peptide on the surface of the tumor cells. Fab 2F1-PE38KDEL which was isolated against HLA-A2/gp100 (G9-280) [89] was labeled with fluorescein isothiocyanate (FITC) and showed internalization in JY cells pulsed with the appropriate gp100-derived G9-280 peptide [59], as shown in Figure 2. These findings indicate that TCR-like antibodies fused to a toxin rapidly internalize after binding to cell surface peptide-MHC complexes and are therefore suitable to use as specific toxin-delivery agents. The TCR-like antibodies fused to PE38KDEL displayed specific cytotoxic activity both in vitro on cells that express natural endogenous differentiation antigens gp100, MART-1, and TARP, and in vivo. All the recombinant immunotoxins analyzed by us showed high binding specificity to antigen-presenting cells (APCs) and melanoma cells expressing the specific peptide/HLA-A201 complex. The immunotoxin 2F1-PE38KEDL had a high rate of internalization (within $30 \mathrm{~min}$ ) and accumulation in the cytosol of the cell (at $6 \mathrm{~h}$ ), where it exerted its function. When tested in vitro, Fab-PE38KDEL immunotoxins killed APCs and melanoma cells in a peptidedependent MHC-restricted manner. Furthermore, CLA12 (that was isolated against HLA-A2/MART)PE38KDEL and 2F1-PE38KDEL had specific antitumor activity in a mouse xenograft model for human melanoma (i.e., they penetrated solid tumors and substantially delayed tumor growth in mice at doses that did not cause animal toxicity). Furthermore, since we constructed immunotoxins against several melanoma epitopes, we explored their concurrent use in an attempt to increase the probability of eradicating tumor cell variants resulting from mutations (e.g., cells that lack the target antigen entirely or express it at levels too low for effective immunotoxin-mediated killing). Indeed, such variants could be eradicated by the combining of two or more immunotoxins recognizing different target antigens [90]. Our results warrant the evaluation of a different class of toxins for melanoma, using a human antibody fragment which is less immunogenic than a mouse antibody and of smaller size, thereby facilitating repeated administration and better tumor penetration. Notably, strategies to reduce the toxicity and the immunogenicity of the toxin, such as a chemical modification with polyethylene glycol [91], will be required to maximize the toxin therapeutic potency in humans. Nonetheless, our data provide a proof-of-concept for the utility of TCR-like antibodies as targeting agents in cancer and form the foundation for further improvement of TCR-like immunotoxins needed to further evaluate their therapeutic potential in more robust in-vivo models. TCR-like immunotoxins, as well as naked TCR-like antibodies that induce apoptosis by direct killing mechanism, may confer clinical benefit in the treatment of immunocompromised patients that may not respond to agents whose mechanisms require a functioning immune system (i.e., ADCC).

\subsection{TCR-Like Antibody-Toxin Fusion Molecules}

TCR-like antibodies have been generated also in a form that is linked with cytokines. Such molecules might target immunomodulatory functions at the site of the tumor or infection. For example, Interferon alpha was used in a fusion protein in which a TCR-like antibody targeting hepatitis B virus infected cells was used to target the cytokine to the infected cells in induce immunomodulation and destruction of the virally-infected cells [92]. 


\section{Outstanding Research Questions and Future Clinical Development of TCR-Like Antibodies}

The recent advances in generating TCR-like antibodies resulted in a growing repertoire of antibodies with specificities directed to a large variety of MHC class I-peptide complexes. These antibodies constitute valuable tool in molecular immunology for studying antigen presentation and developing new immunotherapy agents. TCR-like antibodies contribute to advance basic research of antigen presentation in various healthy or diseased tissues and cell types including tumours, virusinfected cell, professional APCs, and lymphoid cells. The potential therapeutic and in vivo diagnostic utility of TCR-like antibodies was explored and a proof of concept was demonstrated for several tumor types and infectious diseases. The TCR-like antibody approach is now facing the challenge of transitioning into clinical trials. To this end, a candidate antibody should have a high-affinity and highspecificity binding to the TCR ligand. High affinity, in the low-nanomolar or picomolar range, implies a longer binding time and increased probability for therapeutic cytotoxic endocytosis or induction of immune-effector mechanisms. TCR-like specificity of the antibodies is key for their utility as specific targeting molecules.

A strategy that employs recombinant engineered TCRs $[93,94]$ is now being tested in clinical trials, thus, the utilization of TCR-like antibodies is a matter of time required for pre-clinical development.

The antibody isolation procedure could benefit from a comprehensive specificity characterization carried out by mapping the recognition residues within the peptide and the MHC molecule. This procedure would verify the peptide specific, MHC-restricted, TCR-like binding pattern of the antibody. Cross-reactivity to other self-peptides should be excluded by testing binding to a variety of cells types from a range of tissue origins. Another key factor in optimizing TCR-like antibody therapeutic approach is the choice of target antigen from all the T-cell epitopes expressed on malignant cells (studies with anti-cancer mAbs have demonstrated that high density of membrane epitopes on target cells is correlated with the therapeutic efficiency of these agents. For example, high expression of the pMHC antigen on the surface of tumor cells makes it an attractive target for TCR-like antibody therapy. Thus, studies evaluating expression levels of therapeutically-relevant T-cell epitopes (e.g., the aforementioned study on melanoma T-cell epitopes) can reveal optimal targets for TCR-like antibody therapy. Notably, the TCR-like antibodies are also important tool for such studies alongside proteomic and mass spectrometry methodologies.

TCR-like antibody approaches fit in with the growing field of personalized medicine. These reagents may be used for therapies and diagnostics of HLA-matched patients.

The TCR-like antibodies needs to be tailored in terms of their restricted usage in patients with the appropriate HLA class I profile. Although the information related to tumor and viral epitopes are often limited to epitopes restricted by few HLA class I alleles usually present in Caucasian and no other ethnicities, recent advances in proteomic tools and analysis of HLA peptide repertoires enable the rapid identification of peptides derived from specific antigens and their HLA restriction even to rare alleles. According to HLA allele distributions in the world-wide population, TCR-like antibodies directed to three HLA alleles for a particular peptide cover $>96 \%$ of the world-wide population of a given HLA-peptide complex.

The availability of TCR-like antibodies directed to several epitopes associated with a certain tumour type raise the possibility of a cocktail treatment composed of antibodies recognizing different antigens. 
Furthermore, when tumor biopsies are available, the antibodies treatment can be personalized to match the tumour antigen presentation hierarchy of a certain patient. TCR-like antibodies cocktail treatment strategy may increase the treatment potency and target malignant cell mutants with absence or decreased expression levels of a certain target antigen. Further development of this approach needs to be tested experimentally with the ultimate goal of applying TCR-like antibodies to clinical practice.

\section{References}

1. Boon, T.; van der Bruggen, P. Human tumor antigens recognized by T lymphocytes. J. Exp. Med. 1996, 183, 725-729.

2. Renkvist, N.; Castelli, C.; Robbins, P.F.; Parmiani, G. A listing of human tumor antigens recognized by $\mathrm{T}$ cells. Cancer Immunol. Immunother. 2001, 50, 3-15.

3. Rosenberg, S.A. Progress in human tumour immunology and immunotherapy. Nature 2001, 411, 380-384.

4. Anichini, A.; Maccalli, C.; Mortarini, R.; Salvi, S.; Mazzocchi, A.; Squarcina, P.; Herlyn, M.; Parmiani, G. Melanoma cells and normal melanocytes share antigens recognized by HLA-A2restricted cytotoxic t cell clones from melanoma patients. J. Exp. Med. 1993, 177, 989-998.

5. Coulie, P.G.; Brichard, V.; Van Pel, A.; Wolfel, T.; Schneider, J.; Traversari, C.; Mattei, S.; De Plaen, E.; Lurquin, C.; Szikora, J.P.; et al. A new gene coding for a differentiation antigen recognized by autologous cytolytic T lymphocytes on HLA-A2 melanomas. J. Exp. Med. 1994, 180, 35-42.

6. Shetty, V.; Sinnathamby, G.; Nickens, Z.; Shah, P.; Hafner, J.; Mariello, L.; Kamal, S.; Vlahovic, G.; Lyerly, H.K.; Morse, M.A.; et al. MHC class I-presented lung cancer-associated tumor antigens identified by immunoproteomics analysis are targets for cancer-specific $\mathrm{T}$ cell response. J. Proteomics 2011, 74, 728-743.

7. Wang, Z.; Marincola, F.M.; Rivoltini, L.; Parmiani, G.; Ferrone, S. Selective histocompatibility leukocyte antigen (HLA)-A2 loss caused by aberrant pre-mRNA splicing in 624mel28 melanoma cells. J. Exp. Med. 1999, 190, 205-215.

8. Offringa, R.; van der Burg, S.H.; Ossendorp, F.; Toes, R.E.; Melief, C.J. Design and evaluation of antigen-specific vaccination strategies against cancer. Curr. Opin. Immunol. 2000, 12, 576-582.

9. Nagaraj, S.; Gabrilovich, D.I. Tumor escape mechanism governed by myeloid-derived suppressor cells. Cancer Res. 2008, 68, 2561-2563.

10. Klenerman, P.; Cerundolo, V.; Dunbar, P.R. Tracking T cells with tetramers: New tales from new tools. Nat. Rev. Immunol. 2002, 2, 263-272.

11. Novellino, L.; Castelli, C.; Parmiani, G. A listing of human tumor antigens recognized by T cells: March 2004 update. Cancer Immunol. Immunother. 2005, 54, 187-207.

12. Morin, P.J.; Sparks, A.B.; Korinek, V.; Barker, N.; Clevers, H.; Vogelstein, B.; Kinzler, K.W. Activation of beta-catenin-Tcf signaling in colon cancer by mutations in beta-catenin or APC. Science 1997, 275, 1787-1790.

13. de Vries, T.J.; Fourkour, A.; Wobbes, T.; Verkroost, G.; Ruiter, D.J.; van Muijen, G.N. Heterogeneous expression of immunotherapy candidate proteins gp100, mart-1, and tyrosinase in human melanoma cell lines and in human melanocytic lesions. Cancer Res. 1997, 57, 3223-3229. 
14. Nakada, T.; Noguchi, Y.; Satoh, S.; Ono, T.; Saika, T.; Kurashige, T.; Gnjatic, S.; Ritter, G.; Chen, Y.T.; Stockert, E.; et al. Ny-eso-1 mrna expression and immunogenicity in advanced prostate cancer. Cancer Immun. 2003, 3, 10.

15. Sugita, Y.; Wada, H.; Fujita, S.; Nakata, T.; Sato, S.; Noguchi, Y.; Jungbluth, A.A.; Yamaguchi, M.; Chen, Y.T.; Stockert, E.; et al. Ny-eso-1 expression and immunogenicity in malignant and benign breast tumors. Cancer Res. 2004, 64, 2199-2204.

16. King, C.R.; Kraus, M.H.; Aaronson, S.A. Amplification of a novel v-erbB-related gene in a human mammary carcinoma. Science 1985, 229, 974-976.

17. Schechter, A.L.; Stern, D.F.; Vaidyanathan, L.; Decker, S.J.; Drebin, J.A.; Greene, M.I.; Weinberg, R.A. The neu oncogene: An erb-B-related gene encoding a 185,000-Mr tumour antigen. Nature 1984, 312, 513-516.

18. Esche, C.; Shurin, M.R.; Lotze, M.T. The use of dendritic cells for cancer vaccination. Curr. Opin. Mol. Ther. 1999, 1, 72-81.

19. Kugler, A.; Stuhler, G.; Walden, P.; Zoller, G.; Zobywalski, A.; Brossart, P.; Trefzer, U.; Ullrich, S.; Muller, C.A.; Becker, V.; et al. Regression of human metastatic renal cell carcinoma after vaccination with tumor cell-dendritic cell hybrids. Nat. Med. 2000, 6, 332-336.

20. Andersen, P.S.; Stryhn, A.; Hansen, B.E.; Fugger, L.; Engberg, J.; Buus, S. A recombinant antibody with the antigen-specific, major histocompatibility complex-restricted specificity of $\mathrm{T}$ cells. Proc. Natl. Acad. Sci. USA 1996, 93, 1820-1824.

21. Altman, J.D.; Moss, P.A.; Goulder, P.J.; Barouch, D.H.; McHeyzer-Williams, M.G.; Bell, J.I.; McMichael, A.J.; Davis, M.M. Phenotypic analysis of antigen-specific T lymphocytes. Science 1996, 274, 94-96.

22. Denkberg, G.; Cohen, C.J.; Segal, D.; Kirkin, A.F.; Reiter, Y. Recombinant human single-chain MHC-peptide complexes made from E. coli by in vitro refolding: Functional single-chain MHC-peptide complexes and tetramers with tumor associated antigens. Eur. J. Immunol. 2000, $30,3522-3532$.

23. Garboczi, D.N.; Hung, D.T.; Wiley, D.C. HLA-A2-peptide complexes: Refolding and crystallization of molecules expressed in Escherichia coli and complexed with single antigenic peptides. Proc. Natl. Acad. Sci. USA 1992, 89, 3429-3433.

24. Denkberg, G.; Cohen, C.J.; Reiter, Y. Critical role for CD8 in binding of mhe tetramers to TCR: CD8 antibodies block specific binding of human tumor-specific MHC-peptide tetramers to TCR. J. Immunol. 2001, 167, 270-276.

25. Abastado, J.P.; Darche, S.; Jouin, H.; Delarbre, C.; Gachelin, G.; Kourilsky, P. A monoclonal antibody recognizes a subset of the H-2Dd mouse major class I antigens. Res. Immunol. 1989, 140, 581-594.

26. Aharoni, R.; Teitelbaum, D.; Arnon, R.; Puri, J. Immunomodulation of experimental allergic encephalomyelitis by antibodies to the antigen-Ia complex. Nature 1991, 351, 147-150.

27. Biddison, W.E.; Turner, R.V.; Gagnon, S.J.; Lev, A.; Cohen, C.J.; Reiter, Y. Tax and M1 peptide/HLA-A2-specific fabs and $\mathrm{T}$ cell receptors recognize nonidentical structural features on peptide/HLA-A2 complexes. J. Immunol. 2003, 171, 3064-3074.

28. Catipovic, B.; Dal Porto, J.; Mage, M.; Johansen, T.E.; Schneck, J.P. Major histocompatibility complex conformational epitopes are peptide specific. J. Exp. Med. 1992, 176, 1611-1618. 
29. Chames, P.; Hufton, S.E.; Coulie, P.G.; Uchanska-Ziegler, B.; Hoogenboom, H.R. Direct selection of a human antibody fragment directed against the tumor T-cell epitope HLA-A1MAGE-A1 from a nonimmunized phage-Fab library. Proc. Natl. Acad. Sci. USA 2000, 97, 7969-7974.

30. Cohen, C.J.; Denkberg, G.; Lev, A.; Epel, M.; Reiter, Y. Recombinant antibodies with MHCrestricted, peptide-specific, T-cell receptor-like specificity: New tools to study antigen presentation and TCR-peptide-MHC interactions. J. Mol. Recognit. 2003, 16, 324-332.

31. Cohen, C.J.; Hoffmann, N.; Farago, M.; Hoogenboom, H.R.; Eisenbach, L.; Reiter, Y. Direct detection and quantitation of a distinct T-cell epitope derived from tumor-specific epithelial cellassociated mucin using human recombinant antibodies endowed with the antigen-specific, major histocompatibility complex-restricted specificity of T cells. Cancer Res. 2002, 62, 5835-5844.

32. Cohen, C.J.; Sarig, O.; Yamano, Y.; Tomaru, U.; Jacobson, S.; Reiter, Y. Direct phenotypic analysis of human MHC class I antigen presentation: Visualization, quantitation, and in situ detection of human viral epitopes using peptide-specific, MHC-restricted human recombinant antibodies. J. Immunol. 2003, 170, 4349-4361.

33. Dadaglio, G.; Nelson, C.A.; Deck, M.B.; Petzold, S.J.; Unanue, E.R. Characterization and quantitation of peptide-MHC complexes produced from hen egg lysozyme using a monoclonal antibody. Immunity 1997, 6, 727-738.

34. Day, P.M.; Yewdell, J.W.; Porgador, A.; Germain, R.N.; Bennink, J.R. Direct delivery of exogenous MHC class I molecule-binding oligopeptides to the endoplasmic reticulum of viable cells. Proc. Natl. Acad. Sci. USA 1997, 94, 8064-8069.

35. Denkberg, G.; Cohen, C.J.; Lev, A.; Chames, P.; Hoogenboom, H.R.; Reiter, Y. Direct visualization of distinct $\mathrm{T}$ cell epitopes derived from a melanoma tumor-associated antigen by using human recombinant antibodies with MHC- restricted $\mathrm{T}$ cell receptor-like specificity. Proc. Natl. Acad. Sci. USA 2002, 99, 9421-9426.

36. Froscher, B.G.; Klinman, N.R. Immunization with SV40-transformed cells yields mainly MHCrestricted monoclonal antibodies. J. Exp. Med. 1986, 164, 196-210.

37. Held, G.; Matsuo, M.; Epel, M.; Gnjatic, S.; Ritter, G.; Lee, S.Y.; Tai, T.Y.; Cohen, C.J.; Old, L.J.; Pfreundschuh, M.; et al. Dissecting cytotoxic T cell responses towards the NY-ESO-1 protein by peptide/MHC-specific antibody fragments. Eur. J. Immunol. 2004, 34, 2919-2929.

38. Hogquist, K.A.; Grandea, A.G., 3rd; Bevan, M.J. Peptide variants reveal how antibodies recognize major histocompatibility complex class I. Eur. J. Immunol. 1993, 23, 3028-3036.

39. Krogsgaard, M.; Wucherpfennig, K.W.; Cannella, B.; Hansen, B.E.; Svejgaard, A.; Pyrdol, J.; Ditzel, H.; Raine, C.; Engberg, J.; Fugger, L. Visualization of myelin basic protein (MBP) T cell epitopes in multiple sclerosis lesions using a monoclonal antibody specific for the human histocompatibility leukocyte antigen (HLA)-DR2-MBP 85-99 complex. J. Exp. Med. 2000, 191, 1395-1412.

40. Lev, A.; Denkberg, G.; Cohen, C.J.; Tzukerman, M.; Skorecki, K.L.; Chames, P.; Hoogenboom, H.R.; Reiter, Y. Isolation and characterization of human recombinant antibodies endowed with the antigen-specific, major histocompatibility complex-restricted specificity of T cells directed toward the widely expressed tumor T-cell epitopes of the telomerase catalytic subunit. Cancer Res. 2002, 62, 3184-3194. 
41. Polakova, K.; Plaksin, D.; Chung, D.H.; Belyakov, I.M.; Berzofsky, J.A.; Margulies, D.H. Antibodies directed against the MHC-I molecule H-2Dd complexed with an antigenic peptide: Similarities to a t cell receptor with the same specificity. J. Immunol. 2000, 165, 5703-5712.

42. Porgador, A.; Yewdell, J.W.; Deng, Y.; Bennink, J.R.; Germain, R.N. Localization, quantitation, and in situ detection of specific peptide-MHC class I complexes using a monoclonal antibody. Immunity 1997, 6, 715-726.

43. Reiter, Y.; Di Carlo, A.; Fugger, L.; Engberg, J.; Pastan, I. Peptide-specific killing of antigenpresenting cells by a recombinant antibody-toxin fusion protein targeted to major histocompatibility complex/peptide class I complexes with $\mathrm{T}$ cell receptor-like specificity. Proc. Natl. Acad. Sci. USA 1997, 94, 4631-4636.

44. Rubin, B.; Malissen, B.; Jorgensen, P.N.; Zeuthen, J. Recognition of insulin on MHC-class-IIexpressing 1929 cells by antibody and T cells. Res. Immunol. 1989, 140, 67-74.

45. Tamminen, W.L.; Wraith, D.; Barber, B.H. Searching for MHC-restricted anti-viral antibodies: Antibodies recognizing the nucleoprotein of influenza virus dominate the serological response of c57b1/6 mice to syngeneic influenza-infected cells. Eur. J. Immunol. 1987, 17, 999-1006.

46. Uchanska-Ziegler, B.; Nossner, E.; Schenk, A.; Ziegler, A.; Schendel, D.J. Soluble T cell receptor-like properties of an HLA-B35-specific monoclonal antibody (TU165). Eur. J. Immunol. 1993, 23, 734-738.

47. Wylie, D.E.; Sherman, L.A.; Klinman, N.R. Participation of the major histocompatibility complex in antibody recognition of viral antigens expressed on infected cells. J. Exp. Med. 1982, 155, 403-414.

48. Yamano, Y.; Cohen, C.J.; Takenouchi, N.; Yao, K.; Tomaru, U.; Li, H.C.; Reiter, Y.; Jacobson, S. Increased expression of human t lymphocyte virus type I (HTLV-I) Tax11-19 peptide-human histocompatibility leukocyte antigen A*201 complexes on CD4+ CD25+ T cells detected by peptide-specific, major histocompatibility complex-restricted antibodies in patients with HTLV-Iassociated neurologic disease. J. Exp. Med. 2004, 199, 1367-1377.

49. Zehn, D.; Cohen, C.J.; Reiter, Y.; Walden, P. Extended presentation of specific MHC-peptide complexes by mature dendritic cells compared to other types of antigen-presenting cells. Eur. J. Immunol. 2004, 34, 1551-1560.

50. Zhong, G.; Reis e Sousa, C.; Germain, R.N. Production, specificity, and functionality of monoclonal antibodies to specific peptide-major histocompatibility complex class II complexes formed by processing of exogenous protein. Proc. Natl. Acad. Sci. USA 1997, 94, 13856-13861.

51. Benhar, I. Biotechnological applications of phage and cell display. Biotechnol. Adv. 2001, 19, 1-33.

52. de Haard, H.J.; van Neer, N.; Reurs, A.; Hufton, S.E.; Roovers, R.C.; Henderikx, P.; de Bruine, A.P.; Arends, J.W.; Hoogenboom, H.R. A large non-immunized human Fab fragment phage library that permits rapid isolation and kinetic analysis of high affinity antibodies. J. Biol. Chem. 1999, 274, 18218-18230.

53. Griffiths, A.D.; Williams, S.C.; Hartley, O.; Tomlinson, I.M.; Waterhouse, P.; Crosby, W.L.; Kontermann, R.E.; Jones, P.T.; Low, N.M.; Allison, T.J.; et al. Isolation of high affinity human antibodies directly from large synthetic repertoires. EMBO J. 1994, 13, 3245-3260.

54. Hoogenboom, H.R. Selecting and screening recombinant antibody libraries. Nat. Biotech. 2005, $23,1105-1116$. 
55. Chames, P.; Willemsen, R.A.; Rojas, G.; Dieckmann, D.; Rem, L.; Schuler, G.; Bolhuis, R.L.; Hoogenboom, H.R. TCR-like human antibodies expressed on human CTLs mediate antibody affinity-dependent cytolytic activity. J. Immunol. 2002, 169, 1110-1118.

56. Stewart-Jones, G.; Wadle, A.; Hombach, A.; Shenderov, E.; Held, G.; Fischer, E.; Kleber, S.; Nuber, N.; Stenner-Liewen, F.; Bauer, S.; et al. Rational development of high-affinity T-cell receptor-like antibodies. Proc. Natl. Acad. Sci. USA 2009, 106, 5784-5788.

57. Michaeli, Y.; Denkberg, G.; Sinik, K.; Lantzy, L.; Chih-Sheng, C.; Beauverd, C.; Ziv, T.; Romero, P.; Reiter, Y. Expression hierarchy of T cell epitopes from melanoma differentiation antigens: Unexpected high level presentation of tyrosinase-HLA-A2 complexes revealed by peptide-specific, MHC-restricted, TCR-like antibodies. J. Immunol. 2009, 182, 6328-6341.

58. Denkberg, G.; Klechevsky, E.; Reiter, Y. Modification of a tumor-derived peptide at an HLA-A2 anchor residue can alter the conformation of the MHC-peptide complex: Probing with TCR-like recombinant antibodies. J. Immunol. 2002, 169, 4399-4407.

59. Klechevsky, E.; Gallegos, M.; Denkberg, G.; Palucka, K.; Banchereau, J.; Cohen, C.; Reiter, Y. Antitumor activity of immunotoxins with $\mathrm{T}$-cell receptor-like specificity against human melanoma xenografts. Cancer Res. 2008, 68, 6360-6367.

60. Cloutier, S.M.; Couty, S.; Terskikh, A.; Marguerat, L.; Crivelli, V.; Pugnieres, M.; Mani, J.C.; Leisinger, H.J.; Mach, J.P.; Deperthes, D. Streptabody, a high avidity molecule made by tetramerization of in vivo biotinylated, phage display-selected scFV fragments on streptavidin. Mol. Immunol. 2000, 37, 1067-1077.

61. Makler, O.; Oved, K.; Netzer, N.; Wolf, D.; Reiter, Y. Direct visualization of the dynamics of antigen presentation in human cells infected with cytomegalovirus revealed by antibodies mimicking TCR specificity. Eur. J. Immunol. 2010, 40, 1552-1565.

62. Denkberg, G.; Lev, A.; Eisenbach, L.; Benhar, I.; Reiter, Y. Selective targeting of melanoma and APCs using a recombinant antibody with TCR-like specificity directed toward a melanoma differentiation antigen. J. Immunol. 2003, 171, 2197-2207.

63. Sergeeva, A.; Alatrash, G.; He, H.; Ruisaard, K.; Lu, S.; Wygant, J.; McIntyre, B.W.; Ma, Q.; Li, D.; St John, L.; et al. An anti-PR1/HLA-A2 T-cell receptor-like antibody mediates complementdependent cytotoxicity against acute myeloid leukemia progenitor cells. Blood. 2011, 117, 4262-4272.

64. Wittman, V.P.; Woodburn, D.; Nguyen, T.; Neethling, F.A.; Wright, S.; Weidanz, J.A. Antibody targeting to a class I MHC-peptide epitope promotes tumor cell death. J. Immunol. 2006, 177, 4187-4195.

65. Sastry, K.S.R.; Too, C.T.; Kaur, K.; Gehring, A.J.; Low, L.; Javiad, A.; Pollicino, T.; Li, L.; Kennedy, P.T.F.; Lopatin, U.; et al. Targeting hepatitis B virus-infected cells with a T-cell receptor-like antibody. J. Virol. 2011, 85, 1935-1942.

66. Herschhorn, A.; Marasco, W.A.; Hizi, A. Antibodies and lentiviruses that specifically recognize a $\mathrm{T}$ cell epitope derived from HIV-1 Nef protein and presented by HLA-C. J. Immunol. 2010, 185, $7623-7632$. 
67. Bernardeau, K.G.S.; David, G.; Ruellan, A.L.; Devys, A.; Barbet, J.; Bonneville, M.; Chérel, M.; Davodeau, F. Assessment of CD8 involvement in T cell clone avidity by direct measurement of HLA-A2/MAGE3 complex density using a high-affinity TCR like monoclonal antibody. Eur. J. Immunol. 2005, 35, 2864-2875.

68. Verma, B.; Neethling, F.A.; Caseltine, S.; Fabrizio, G.; Largo, S.; Duty, J.A.; Tabaczewski, P.; Weidanz, J.A. TCR mimic monoclonal antibody targets a specific peptide/hla class I complex and significantly impedes tumor growth in vivo using breast cancer models. J. Immunol. 2010, 184, 2156-2165.

69. Bronner, V.; Denkberg, G.; Peled, M.; Elbaz, Y.; Zahavi, E.; Kasoto, H.; Reiter, Y.; Notcovich, A.; Bravman, T. Therapeutic antibodies: Discovery and development using the ProteOn XPR3 biosensor interaction array system. Anal. Biochem. 2010, 406, 147-156.

70. Weiner, L.M.; Surana, R.; Wang, S. Monoclonal antibodies: Versatile platforms for cancer immunotherapy. Nat. Rev. Immunol. 2010, 10, 317-327.

71. Dahan, R.; Reiter, Y. T-cell-receptor-like antibodies-Generation, function and applications. Expert Rev. Mol. Med. 2012, 14, e6.

72. Cartron, G.; Dacheux, L.; Salles, G.; Solal-Celigny, P.; Bardos, P.; Colombat, P.; Watier, H. Therapeutic activity of humanized anti-CD20 monoclonal antibody and polymorphism in IgG Fc receptor FcgammaRIIIa gene. Blood 2002, 99, 754-758.

73. Clynes, R.A.; Towers, T.L.; Presta, L.G.; Ravetch, J.V. Inhibitory Fc receptors modulate in vivo cytotoxicity against tumor targets. Nat. Med. 2000, 6, 443-446.

74. Edwards, M.S.; Chadda, S.D.; Zhao, Z.; Barber, B.L.; Sykes, D.P. A systematic review of treatment guidelines for metastatic colorectal cancer. Colorectal Dis. 2012, 14, e31-e47.

75. Kreitman, R.J. Immunotoxins for targeted cancer therapy. AAPS J. 2006, 8, E532-E551.

76. Pastan, I.; Hassan, R.; FitzGerald, D.J.; Kreitman, R.J. Immunotoxin treatment of cancer. Annu. Rev. Med. 2007, 58, 221-237.

77. Shapira, A.; Benhar, I. Toxin-based therapeutic approaches. Toxins 2010, 2, 2519-2583.

78. Litvak-Greenfeld, D.; Benhar, I. Risks and untoward toxicities of antibody-based immunoconjugates. Adv. Drug Deliv. Rev. 2012, 64, 1782-1799.

79. Falnes, P.O.; Sandvig, K. Penetration of protein toxins into cells. Curr. Opin. Cell Biol. 2000, 12, 407-413.

80. Menestrina, G.; Schiavo, G.; Montecucco, C. Molecular mechanisms of action of bacterial protein toxins. Mol. Aspects Med. 1994, 15, 79-193.

81. Chiron, M.F.; Fryling, C.M.; FitzGerald, D. Furin-mediated cleavage of pseudomonas exotoxinderived chimeric toxins. J. Biol. Chem. 1997, 272, 31707-31711.

82. Williams, D.P.; Wen, Z.; Watson, R.S.; Boyd, J.; Strom, T.B.; Murphy, J.R. Cellular processing of the interleukin-2 fusion toxin DAB486-IL-2 and efficient delivery of diphtheria fragment A to the cytosol of target cells requires Arg194. J. Biol. Chem. 1990, 265, 20673-20677.

83. Carroll, S.F.; Collier, R.J. Active site of pseudomonas aeruginosa exotoxin a. Glutamic acid 553 is photolabeled by nad and shows functional homology with glutamic acid 148 of diphtheria toxin. J. Biol. Chem. 1987, 262, 8707-8711. 
84. Van Ness, B.G.; Howard, J.B.; Bodley, J.W. Adp-ribosylation of elongation factor 2 by diphtheria toxin. Isolation and properties of the novel ribosyl-amino acid and its hydrolysis products. J. Biol. Chem. 1980, 255, 10717-10720.

85. Kreitman, R.J. Recombinant immunotoxins containing truncated bacterial toxins for the treatment of hematologic malignancies. BioDrugs 2009, 23, 1-13.

86. Seetharam, S.; Chaudhary, V.K.; FitzGerald, D.; Pastan, I. Increased cytotoxic activity of pseudomonas exotoxin and two chimeric toxins ending in KDEL. J. Biol. Chem. 1991, 266, 17376-17381.

87. Denkberg, G.; Lev, A.; Eisenbach, L.; Benhar, I.; Reiter, Y. Selective targeting of melanoma and APCs using a recombinant antibody with TCR-like specificity directed toward a melanoma differentiation antigen. J. Immunol. 2003, 171, 2197-2207.

88. Epel, M.; Carmi, I.; Soueid-Baumgarten, S.; Oh, S.K.; Bera, T.; Pastan, I.; Berzofsky, J.; Reiter, Y. Targeting TARP, a novel breast and prostate tumor-associated antigen, with $\mathrm{T}$ cell receptor-like human recombinant antibodies. Eur. J. Immunol. 2008, 38, 1706-1720.

89. Cohen, C.J.; Denkberg, G.; Segal, D.; Reiter, Y. Generation of recombinant immunotoxins for specific targeting of tumor-related peptides presented by MHC molecules. Methods Mol. Biol. 2003, 207, 269-282.

90. Ghetie, M.A.; Tucker, K.; Richardson, J.; Uhr, J.W.; Vitetta, E.S. The antitumor activity of an anti-CD22 immunotoxin in scid mice with disseminated Daudi lymphoma is enhanced by either an anti-CD19 antibody or an anti-CD19 immunotoxin. Blood 1992, 80, 2315-2320.

91. Tsutsumi, Y.; Onda, M.; Nagata, S.; Lee, B.; Kreitman, R.J.; Pastan, I. Site-specific chemical modification with polyethylene glycol of recombinant immunotoxin anti-Tac(Fv)-PE38 (LMB-2) improves antitumor activity and reduces animal toxicity and immunogenicity. Proc. Natl. Acad. Sci. USA 2000, 97, 8548-8553.

92. Ji, C.; Sastry, K.S.; Tiefenthaler, G.; Cano, J.; Tang, T.; Ho, Z.Z.; Teoh, D.; Bohini, S.; Chen, A.; Sankuratri, S.; et al. Targeted delivery of interferon- $\alpha$ to hepatitis B virus-infected cells using T-cell receptor-like antibodies. Hepatology 2012, 56, 2027-2038.

93. Liddy, N.; Bossi, G.; Adams, K.J.; Lissina, A.; Mahon, T.M.; Hassan, N.J.; Gavarret, J.; Bianchi, F.C.; Pumphrey, N.J.; Ladell, K.; et al. Monoclonal TCR-redirected tumor cell killing. Nat. Med. 2012, 18, 980-987.

94. Molloy, P.E.; Sewell, A.K.; Jakobsen, B.K. Soluble T cell receptors: Novel immunotherapies. Curr. Opin. Pharmacol. 2005, 5, 438-443.

(C) 2013 by the authors; licensee MDPI, Basel, Switzerland. This article is an open access article distributed under the terms and conditions of the Creative Commons Attribution license (http://creativecommons.org/licenses/by/3.0/). 